\title{
Why Johnny Can't Unsubscribe: Barriers to Stopping Unwanted Email
}

\author{
Jayati Dev \\ Luddy School of Informatics, \\ Computing, and Engineering \\ Indiana University \\ Bloomington \\ jdev@iu.edu
}

\author{
Emilee Rader \\ Department of Media and \\ Information \\ Michigan State University \\ emilee@msu.edu
}

\author{
Sameer Patil \\ Luddy School of Informatics, \\ Computing, and Engineering \\ Indiana University \\ Bloomington \\ patil@indiana.edu
}

\begin{abstract}
A large proportion of email messages in an average Internet user's inbox are unwanted commercial messages from mailing lists, bots, and so on. Although such messages often include instructions to unsubscribe, people still struggle with stopping unwanted email. We investigated the user experience of unsubscribing from unwanted email messages by recruiting 18 individuals for via a lab study followed by semi-structured interviews. Based on unsubscribing practices of the study participants, we synthesized eight common unsubscription mechanisms and identified the corresponding user experience challenges. We further uncovered alternative practices aimed at circumventing the need to unsubscribe. Our findings reveal frustration with the prevailing options for limiting access to the self by managing email boundaries. We apply our insight to offer design suggestions that could help commercial providers improve the user experience of unsubscribing and provide users more control over the email they receive.
\end{abstract}

\section{Author Keywords}

Unsubscribing; Unwanted email; Marketing email; Newsletters; Mailing lists; Opt-out; Boundary management; Privacy

\section{CCS Concepts}

-Security and privacy $\rightarrow$ Usability in security and privacy; •Human-centered computing $\rightarrow$ Empirical studies in HCI;

\section{INTRODUCTION}

In recent years, email has shifted from a medium for interpersonal communication to a channel used extensively for communication between individuals and institutions. As people have begun using other kinds of messaging services to communicate with friends, family, and colleagues, email has become dominated by messages sent from mailing lists, businesses, political candidates, doctors' offices, and social websites, among

This work is licensed under a Creative Commons Attribution International 4.0 License. CHI'20, April 25-30, 2020, Honolulu, HI, USA. Copyright is held by the author/owner(s). (C)(1) ACM ISBN 978-1-4503-6708-0/20/04

http://dx.doi.org/10.1145/3313831.3376165 others [2]. One study estimated that more than $90 \%$ of nonspam email consists of automated messages from institutional senders [4]. As a result, the overall volume of email continues to increase. In 2018, over 250 billion email messages were sent and received each day [10]. Nearly forty-five percent of these messages were deemed spam ${ }^{1}$. Although effective filters prevent most spam from making it to inboxes, people still report receiving large amounts of unwanted email [9]. Yet, email systems currently provide few technical mechanisms to help manage or prevent unwanted email.

Unsubscribing as a way to manage unwanted email is different from other strategies, such as leaving messages unread or mass-deleting unwanted email [4], because it involves explicitly signalling to the sender that the email is unwanted. Users must rely on the institutions that send them unwanted email to provide a way to opt out and, subsequently, to honor their wishes by not sending such email in the future. While many countries have enacted legislation requiring that institutions allow people to opt out of marketing communications (e.g., the CAN-SPAM Act of 2003 in the United States [18]), these regulations may not ensure that opt-out options are obvious or that people are successful in achieving their goal of unsubscribing.

To understand the challenges related to unsubscribing from unwanted email, we conducted a study in which we observed people while they tried to unsubscribe and interviewed them about the experience. Unsubscribe mechanisms have the potential to be an important tool for users to engage in boundary management to prevent unwanted email from reaching them. In reality, we found that study participants faced many problems and barriers when they tried to unsubscribe.

Based on the email messages encountered during the study, we contribute representations of the process flows of a variety of unsubscription mechanisms and describe corresponding challenges encountered when attempting to unsubscribe. We further identify the reasons people choose to unsubscribe from unwanted email (or not) and report the alternatives adopted by some participants to circumvent the process of unsubscribing. We connect the findings of the study to theories that define privacy as a process of boundary regulation in which people aim to manage their levels of accessibility to others [1]. Finally, we offer design recommendations for commercial senders and

\footnotetext{
${ }_{1}$ https://www . spamlaws . com/spam-stats .html
} 
email providers that could help improve the user experience of unsubscribing from unwanted email.

\section{BACKGROUND}

Our work is motivated by the overload created by ever increasing volumes of incoming email, requiring users to develop various coping strategies.

\section{Users are Overwhelmed by Institutional Email}

Research on email use has identified a major shift in recent years, from interpersonal communication to institutional communication. People rarely communicate with friends and family via email anymore. Rather, email is used mostly for corresponding with coworkers and receiving mass automated email from institutions or commercial senders $[2,4]$. In an interview study, Bentley et al. [2] found that users were subscribed to 93 different email lists, on average, and more than two thirds reported using email mainly for receiving information about deals and coupons.

The shift in email use has meant a corresponding shift in the nature of email overload. Whittaker and Sidner defined email overload as the use of email for tasks other than communication, like managing to-dos or information management [21]. However, email overload today typically results from receiving an overwhelming amount of automated messages. One study estimated that more than $90 \%$ of non-spam email consists of automated messages from institutional senders [4]. Another study found that $95 \%$ of such email was subscription-based [7]. The large amount of institutional email is causing people to feel more and more overwhelmed [2,9].

Institutional or commercial email may be overwhelming and unwanted, but it is not what users consider spam [9]. People tend to use the term "spam" to refer to unsolicited or unwanted email that is potentially harmful or malicious [16]. Spam filters cannot effectively deal with such non-spam unwanted email because there is less agreement across users about how it should be handled. People actually want to receive some of the commercial or marketing email $[6,19]$, thus creating too much inconsistency in preferences that prevents automated spam filtering solutions to be broadly effective in tackling non-spam unwanted email.

\section{Current Solutions are Ineffective}

When given an effective mechanism, people are known to assert control over the ability of other parties to contact them. When the United States instituted a national Do-Not-Call registry in 2003 to prevent unsolicited telemarketing phone calls, more than 100 million telephone numbers were registered within two years [12]. An empirical analysis of registrations showed that telemarketers increased the number of calls to those not on the list, assuming that not signing up for the registry indicated that these individuals were more receptive to telemarketing calls. Consequently, even more people registered to avoid the increased telemarketing resulting from not being in the registry [8].

In many countries, there is legislation in place to help prevent unwanted commercial email. The U.S. CAN-SPAM Act of
2003 [18] mandates that commercial senders may send unsolicited email to anyone who has not explicitly opted out, provided the email does not involve deception and the sender honors requests to opt out [17]. However, few people currently attempt to exercise opt-out options [3]. Usability problems with unsubscribe mechanisms in email messages may be partly responsible for the under-utilization of unsubscribing. The Nielsen Norman Group reported that senders often hide unsubscribe options in links that are hard for users to see and require going through many confusing steps in order to unsubscribe [6]. The report indicates that senders of subscriptionbased email may be intentionally making it difficult for people to unsubscribe, not recognizing the potentially negative impact on user trust created by these unsubscription barriers.

\section{Users Must Cope with Unwanted Email}

Anyone can send email to someone simply by knowing the person's email address. Recipients cannot prevent senders from contacting them, thus making it challenging to avoid unwanted email. The email protocol does not provide a mechanism to prevent the receipt of certain messages and not others [7]. In the absence of effective mechanisms to prevent senders from barraging recipients with unwanted institutional or commercial email, people typically cope with the overload by not opening most of the email messages they receive, leaving them unread [2]. A large-scale analysis of email inboxes found that, on average, $85 \%$ of email messages are never read [4], and a similar study reported that nearly $90 \%$ of deleted email messages were not read before deletion [7]. Some users are so overwhelmed by unwanted email that they abandon their email accounts altogether and start fresh with new accounts [2].

Altman [1] defined privacy as "selective control of access to the self." The ability to manage email boundaries by preventing unwanted communication from others is an important part of such control. While researchers have applied Altman's theoretical characterization to information/data privacy, his original conceptualization describes privacy as "an interpersonal boundary control process" [1]. In addition, Petronio [15] argues that people need the ability to negotiate appropriate communication boundaries in cooperation with other individuals and institutions and the assurance that those boundaries will be respected. Boundary coordination is an important aspect of protecting oneself from threats to one's autonomy through manipulation by others [14].

Email users need better ways to define boundaries and exert control over the overwhelming amount of incoming commercial email. While research has examined opt-out practices for online behavioral advertising [13], there has not yet been a similar investigation of opting out of email by unsubscribing. We fill this gap with an in-depth study of why and how people unsubscribe (or not) from email messages they consider unwanted. Understanding the various facets of unsubscription practices, workarounds, and motivations would help people navigate their email better in terms of the content they find relevant. Focusing on people's actions and thoughts while unsubscribing can provide a way for companies to design more accessible interfaces, implement alternative mechanisms for unsubscribing and instill trust in their consumer base. 
Table 1: Characteristics of the participants in the sample.

\begin{tabular}{rcccc}
\hline & Age & & Daily Email & \\
ID & Group & Gender & Volume & Field \\
\hline 1 & $20-29$ & Non-binary & $26-50$ & Psychology \\
2 & $60-69$ & Female & $76-100$ & Informatics \\
3 & $20-29$ & Female & $>100$ & Cybersecurity \\
4 & $30-39$ & Female & $10-25$ & Music Education \\
5 & $30-39$ & Male & $>100$ & Communication \\
6 & $60-69$ & Female & $26-50$ & \\
7 & $30-39$ & Male & $10-25$ & Adult Education \\
8 & $60-69$ & Male & $26-50$ & \\
9 & $20-29$ & Female & $51-75$ & Sociology/Spanish \\
10 & $40-49$ & Male & $>100$ & \\
11 & $20-29$ & Male & $10-25$ & Informatics \\
12 & $20-29$ & Male & $10-25$ & Neuroscience/Biology \\
13 & $20-29$ & Female & $>100$ & Counseling Psychology \\
14 & $20-29$ & Male & $10-25$ & Liberal Studies \\
15 & $30-39$ & No answer & $51-75$ & Music \\
16 & $40-49$ & Male & $>100$ & Public Health \\
17 & $20-29$ & Female & $10-25$ & Env. Science \\
18 & $20-29$ & Female & $10-25$ & Marketing \\
\hline
\end{tabular}

\section{METHOD}

We investigated how users unsubscribe from unwanted email by conducting an in-person study.

\section{Recruitment and Screening}

We recruited participants during the Spring and Summer of 2019 via flyers posted at Indiana University and around town in Bloomington, Indiana. In addition, we posted advertisements to online forums and mailing lists. The flyers and advertisements included a link to an online screening questionnaire. Anyone who encountered the link could fill out the screening questionnaire. Anyone at least 18 years old and able to attend an in-person lab study at Indiana University was eligible to participate. Since we were studying individual preferences that might vary across cultures, we recruited only those who had resided in the United States for at least five years in order to ensure sufficient cultural homogeneity in the sample. Prior to the study, we asked those selected for participation to create a new folder in their email applications and save at least 10 unwanted email messages received after we contacted them. We further instructed these individuals not to try to unsubscribe from these email messages before the study.

\section{Participants}

Eighteen people participated in the study. Table 1 provides an overview of the participant characteristics. Apart from one person who identified as non-binary and one who did not provide their gender, participants were equally split between men and women. One participant was partially visually impaired. Participant ages ranged from 22 to 64 years with half being students 20-29 years old. Thirteen participants were undergraduate or graduate students from a diversity of backgrounds in terms of fields of study. Two participants were university staff members, and the remaining three participants were residents of Bloomington not affiliated with Indiana University. This diversity of characteristics allowed us to identify aspects of unsubscribing mechanisms and behavior that are common across people who may use email in diverse ways. We continued collecting data until we reached a reasonable number of participants for a homogeneous sample based on the guidelines of Guest et al. [11] and began to reach saturation in terms of encountering similar unsubscribing behavior, motivations, and barriers compared with those of earlier participants.

\section{Study Session}

Upon reviewing the study information sheet and consenting to participate, we asked participants to login to their email accounts using a lab computer. Next, we asked them to think aloud while trying to unsubscribe from the unwanted email messages they had been asked to save prior to the session. Thinking aloud has been shown to be useful for collecting data about people's thoughts while performing tasks [5]. We did not provide guidance or specific definitions of 'unsubscribing' or help the participants choose specific email messages for the unsubscribing task. The protocol allowed us to observe difficulties with the process of unsubscribing and understand the factors that caused confusion and frustration. After finishing with the saved unwanted email, those who agreed were asked to attempt unsubscribing from email messages in their spam or junk folders. We ensured that no one clicked on an email message that appeared to be malicious based on the the sender or the subject. Each participant unsubscribed from between 5 and 15 email messages, forming a corpus of 171 unsubscribe attempts across a variety of email types.

At the end of the session, we conducted a short semi-structured interview with the participants to understand their motivations and behavior. The interview protocol consisted of general questions about unsubscribing practices, task-specific questions relating to unsubscribing choices and behavior, and background questions about Internet-related practices. We asked participants why they saved certain email messages for the study to learn how they separated desired and unwanted email.

With permission from the participants, we screen recorded the study sessions and audio recorded the post-study interviews for transcription and analysis. The study lasted about 45-60 minutes per session. At the end, we asked participants to log out of their email accounts and cleared all cookies on the lab computer used for the study. We provided participation compensation of U.S. \$10. All study procedures were reviewed and approved by the Institutional Review Boards (IRBs) of Indiana University and Michigan State University. (See supplementary materials for the study protocol and interview questions.)

\section{Analysis}

From the screen recordings of the study sessions, we wrote a description of the process of unsubscribing from when the participant clicked the unsubscribe or update preference link, to unsubscription confirmation. These descriptions were then combined and summarized based on similarities across participants to form eight major mechanisms of unsubscribing. For example, any unsubscribing process that involved a subscription center (a web page with options for subscribing, email frequency preferences, and unsubscribing) was combined into a single mechanism (see Figure $3 a$ ). 
We began by coding the transcripts of the first 12 study sessions. We focused first on the parts of the unsubscribing tasks where participants mentioned having trouble unsubscribing. We identified challenges and frustrations based on participant statements indicating surprise, confusion, or uncertainty. We then analyzed the interview transcripts using an iterative inductive approach involving open coding, pattern development, and aggregation of responses by themes. In the first round of coding, we focused on broad themes related to types of unsubscribing actions and beliefs about those actions. We formed a codebook of higher-level themes synthesized from the first 12 participant sessions. We used the codebook to analyze the transcripts of the remaining six sessions. Codes that appeared only once were excluded from the analysis. Additional themes that emerged from the remaining six sessions were were incorporated into the coding scheme as long as they were present in more than one of the six study sessions.

We then made a pass through the entire data, aggregating responses by themes across participants. The grouping of themes used a theoretical lens grounded in Altman's [1] characterization of privacy as an "interpersonal boundary control process," and Petronio's [15] theory of Communication Privacy Management. As we have argued, unsubscribing is one way that users can establish a boundary between themselves and senders of unwanted email. We brought this perspective to bear by grouping emergent themes based on boundary expectations, boundary enforcement, and boundary turbulence (i.e., poor boundary coordination between email senders and recipients).

\section{Limitations}

Our analysis produced a summary of the unsubscription mechanisms that were commonly encountered by participants during our study. However, there might be unsubscribing mechanisms that we did not encounter. It should be noted that our focus was on email delivered to personal (i.e., non-work) email inboxes. While work email may likely contain lower proportions of unwanted messages, the nature of the unsubscribing considerations are identical regardless of whether unwanted email is sent to personal or work email addresses. Although we believe that our approach is suitable for yielding rich detail on unsubscribing from unwanted email, our findings are necessarily limited by the convenience sample. The sample is homogeneous as all participants are from the United States and connected to a large public university. Moreover, the sample contains a disproportionately large percentage of students and young individuals who are likely more knowledgeable about technology than the general population. The challenges faced by the participants despite higher likelihood of being technically savvy suggest that the situation for the general population is likely to be worse. Although online sessions with remote participants might have helped us solicit a broader sample, we chose an in-person approach to allow finer observations and detailed follow-up questions.

\section{FINDINGS}

Our analysis uncovered commonly encountered unsubscribing processes with poor user experience that hindered effective boundary management and created boundary turbulence.

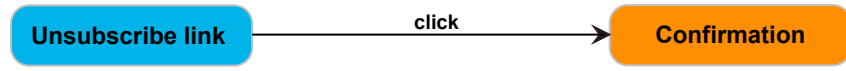

(a) One-Click Unsubscribe: Clicking the unsubscribe link unsubscribes directly without additional clicks.

\begin{tabular}{|c|c|c|}
\hline Unsubscribe link & click & Verification \\
\hline by email provider & & $\downarrow$ submit \\
\hline$\downarrow$ click & & Confirmation \\
\hline $\begin{array}{l}\text { Redirect to } \\
\text { unsubscribe link } \\
\text { within message }\end{array}$ & & Browser pop-up \\
\hline
\end{tabular}

(b) Unsubscribe Link with Confirmation: Unsubscribe link provided by the email provider usually at the top of the email message. The link either opens the landing page linked to the unsubscribe link within the message body or directly confirms the process worked, with a few verification steps. Confirmation is typically presented at the top of the message.

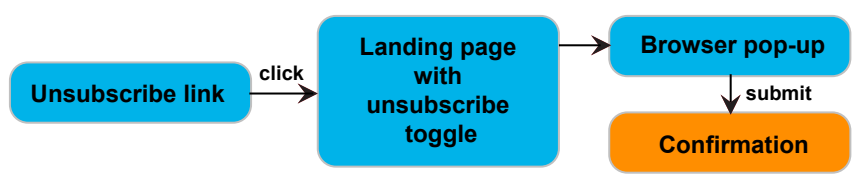

(c) Landing Page with Toggle: Unsubscribe link points to a landing page with an unsubscribe toggle, usually set to opt-in, followed by a confirmation.

Figure 1: Unsubscribing mechanisms reported by participants to be easy and convenient. Blue boxes denote the process or entity encountered during the unsubscribing process, and orange boxes indicate the page confirming the outcome.

\section{Unsubscribing Mechanisms}

We consolidated the processes of unsubscribing followed by the participants into eight different mechanisms presented in Figures 1-3. The processes start at the 'unsubscribe link' (a general reference to the start of the unsubscribe process which did not always visually look like a link) and end with a "confirmation' (the point at which participants believed they had successfully unsubscribed). The path length in each diagram depicts the number of steps necessary to complete the process via the corresponding mechanism. In the cases of mechanisms with multiple steps, whether participants encountered the mechanism fully or partially depended on the characteristics of the respective email messages. Observing participants as they attempted to unsubscribe revealed their preferences, expectations and frustrations regarding the operational implementation of the mechanisms.

\section{Convenient Mechanisms for Unsubscribing}

Figure 1 shows the unsubscribing mechanisms participants found the most straightforward. Figure 1a depicts unsubscribing with a single click on an unsubscribe link which led to a confirmation that the action had been successful; this was the mechanism that participants found the easiest and quickest to follow. The unsubscribe functionality provided by the email provider (GMail, Yahoo, etc.) was the most visible and relieved participants from the burden of scrolling through the email message in order to find an obscure unsubscribe link (see Figure 1b). Participants also found the mechanism that required a toggle switch for the subscription (see Figure 1c) 


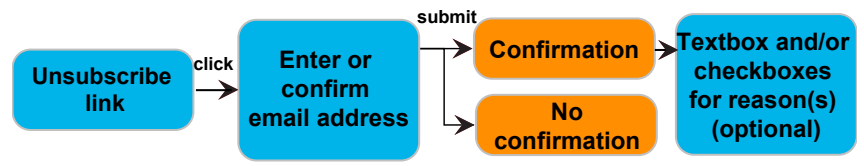

(a) Multiple Confirmation Pages: Unsubscribe link points to a chain of pages, first asking for the email address. Optional feedback is sought at the end. In rare cases, mandatory feedback is needed prior to completing the unsubscribe process.

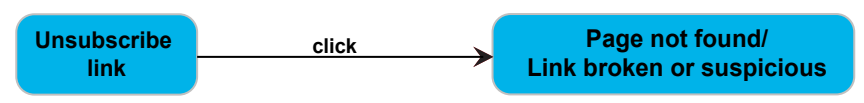

(b) Broken Link: Unsubscribe link leads to a page that is blank, flagged by the browser as suspicious, or broken in some form.

Figure 2: Unsubscribing mechanisms that participants found somewhat inconvenient and difficult. Blue boxes denote the process or entity encountered during the unsubscribing process, and orange boxes indicate the page confirming the outcome.

easy to deal with, even though they found that it unnecessarily introduced the extra step of needing to deal with the switch.

\section{Challenging Mechanisms for Unsubscribing}

Mechanisms presented in Figures 2 and 3 posed participants with various difficulties. In particular, Subscription Center (see Figure 3a), Unsubscribe Through Login (see Figure 3b), and Reply to Unsubscribe (see Figure 3c) presented major barriers to unsubscribing. For instance, no participant was able to unsubscribe successfully when the process required login. Similarly, writing an email message to a person or a mailing list was considered undesirable. P4 avoided the action owing to her familiarity with the members of the lists. Reply to Unsubscribe was, however, rare and occurred only three times in the study. Participants found mechanisms with multiple confirmation pages (see Figure 2a) particularly annoying because of being asked multiple times to enter basic information, such as an email address. Participants were not thrilled when businesses burdened them with inputting information that was already known to the business. In addition, participants did not appreciate being asked for reasons for unsubscribing and believed that the organizations were intentionally trying to make the process more difficult for business purposes.

Most mechanisms ended with a confirmation page. The exceptions were Reply to Unsubscribe (see Figure 3c) and Broken Link (see Figure 2b), neither of which were successfully completed by even a single participant. The confirmations, shown by the orange boxes in Figures 1-3, indicate that the unsubscription process has reached the end. Yet, confirmation pages did not often provide a clear indication that the process had ended. While some confirmation pages explicitly mentioned that the participant had been opted out of future email, many presented ambiguous messages like 'the request is being processed' or 'preferences have been saved.'

With the exception of 2 cases out of 171, none of the confirmation messages assured participants that they would continue to receive email messages pertaining to orders and important

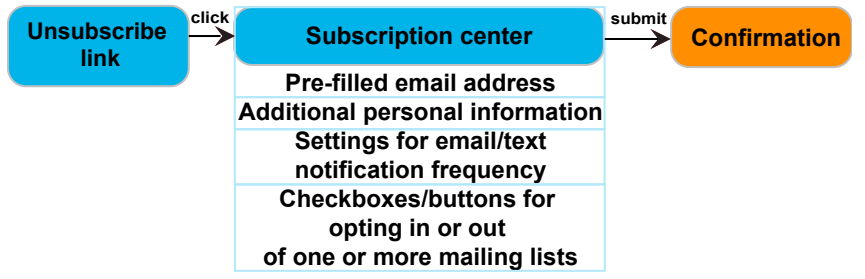

(a) Subscription Center: Clicking unsubscribe link brings up a subscription center page containing parts or all of the elements shown in the figure.

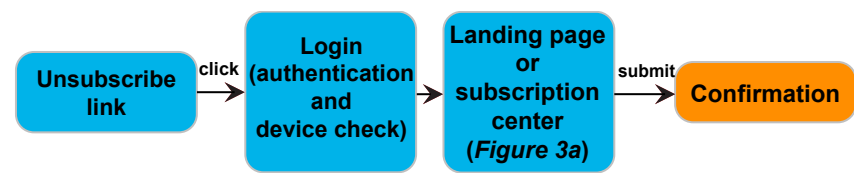

(b) Unsubscribe Through Login: Unsubscribing functionality can be accessed only after logging in. Login may involve additional burden such as multi-factor authentication, device confirmation, etc.

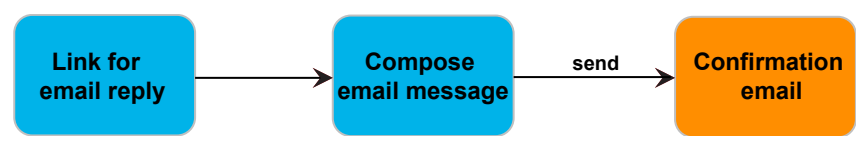

(c) Reply to Unsubscribe: Unsubscribing requires replying to the mailing list or emailing the administrator of the mailing list.

Figure 3: Mechanisms that participants found the most challenging and cumbersome. Blue boxes denote the process or entity encountered during the unsubscribing process, orange boxes indicate the page confirming the outcome

account updates after unsubscribing from promotional content. For example, email messages from Amazon contain unsubscribe links, but participants were not sure if clicking the unsubscribe link in the email messages received from Amazon would unsubscribe them from receiving desired Amazon email messages related to payments and order tracking.

\section{Poor User Experience}

The difficulties faced by the participants in navigating the unsubscribe process often stemmed from the inconsistent and poorly designed user experience across the various mechanisms we described above.

\section{Initiating the Unsubscribing Process}

At the beginning of each unsubscribing task, all participants immediately scrolled to the bottom of the email message and searched for a link labeled 'unsubscribe.' Often, instead of using the commonly expected word 'unsubscribe,' the unsubscribe link was marked with alternate text, such as 'Click Here.' Use of alternate terms required more effort from participants to find the link needed to initiate unsubscription.

Unsubscribe links were not always easy to spot. The links were typically formatted with a tiny font and a color with low contrast against the background and buried among several other links. The low visibility of the links was especially problematic for the visually impaired participant. It took her a long time to scroll through large chunks of text to find hard-tospot unsubscribe links: 
“... my eyes just take a long time to focus on each space. So if it isn't like, sign up, well if I can sign up then I should be able to unsign up, but no... You have to scroll down to find it. Not good." - (P6, Female, 60-69)

Occasionally, email messages were truncated by the email client before the unsubscribe link was visible. P2 required researcher intervention to find the unsubscribe link upon encountering such a situation. P10 mentioned that the truncation made it difficult for him to unsubscribe while accessing email on a mobile device. P12 pointed out that it would be hard for older adults and those with low technical skills to "figure out" how to unsubscribe.

Some unsubscribe mechanisms, such as Unsubscribe Through Login (see Figure 3b), required that participants remember and enter the email address they used to sign up for the unwanted email message in question. When faced with such cases, 5 participants were unable to recall the email address they had used. Participants were greatly frustrated by being asked to recall the applicable email address, especially because they expected the sender to know the email address to which the email was sent. Participants desired that unsubscribing be possible just by "hitting the button" (P7).

\section{Conveying the Unsubscribe Request}

Some landing pages presented options for subscribing as well as unsubscribing, causing confusion among participants as they could not be certain whether they were unsubscribing or signing up for additional content:

"Now I don't know if I'm deleting the subscription or if I'm signing up for something else." - (P5, Male, 30-39)

P9 and P15 experienced similar confusion regarding email messages that included 'subscribe' as well as 'unsubscribe' links, causing them to switch between the two accidentally. P9 conjectured that the message might have been forwarded and hence provided a subscribe link should the user wish to subscribe to the service.

Participants were concerned about unintentionally subscribing for even more unwanted email and co-presence of subscription and unsubscription options required them to devote extra attention and effort to ensure successful unsubscription. P16 demanded additional verification and guarantees as he doubted that the unsubscribe was being honored.

\section{Establishing Successful Unsubscription}

Participants found it difficult to figure out how their request to unsubscribe was handled. Lack of clear and immediate feedback caused participants to wonder if they were successfully unsubscribed. Participants were confused by messages such as 'Your request has been processed' or 'Your preferences have been updated' as the messages did not confirm unsubscription:

"I don't know what that means, maybe I'll still get a few extra emails from them over the next few weeks as they're processing my request?" - (P5, Male, 30-39)

Due to the ambiguous nature of the feedback, P5 and P9 were unsure whether they unsubscribed successfully in cases where the button on the landing page was labeled 'update.' For instance, P9 found it confusing to unsubscribe from SurveyMonkey email messages because the 'unsubscribe' link on the landing pages was broken but the 'update' link worked.

Some confirmation pages mentioned specific lengths of time required for the the unsubscription to take effect. Typical time periods ranged from 24 hours to 7 days. When participants encountered such messages, they were frustrated and puzzled that an automated system would require such a long time to unsubscribe them:

"This could take 24 hours, 72 hours. It's like, it's a computer. I could turn you off right now. You know, so I don't understand why they say that. I mean, I know why they say it, but it looks like it should be automatic. You know, it updates in two minutes, five minutes. I can place an order... within a matter of minutes. I would think unsubscribed could be the same way." - (P2, Female, 60-69)

While two participants believed that the process of unsubscribing had worked for them in the past, others mentioned they felt that they keep getting unwanted email even after trying to unsubscribe multiple times:

"I know that I have unsubscribed from this one before. That's why it's gone to junk because I keep unsubscribing and it keeps sending me email[s]." - (P4, Female, 30-39)

P4 believed that the only way to verify whether unsubscribing worked is to check for future email messages. As a result, she was reluctant to trust the process of unsubscribing.

Some email messages included an unsubscribe link at the top of the email. Such a link was provided by the email provider to allow participants to unsubscribe directly without going through the tedium of the typical unsubscribing mechanisms. With the exception of P17, none of the participants who noticed or used the link believed that it worked:

“... looks like GMail has a thing. I've done this though, and sometimes these don't work" - (P11, Male, 20-29)

While P4 said that "it never works," P9 was curious to try it out during the study session. P9 proceeded to click the "Learn More" link presented by GMail alongside the unsubscribe link, thus discovering that it takes a few days to unsubscribe using the link, which was not intuitive.

\section{Boundary Management for Unwanted Email}

Every email message that arrives in a user's inbox demands attention and action. One of the fundamental characteristics of email is that anyone who has a person's email address can send email messages to that person. Consequently, it can be hard for users to establish boundaries between acceptable and unacceptable email messages. Participants in our study wanted to establish boundaries by preventing some of the incoming email from reaching them. Although participants believed that unsubscribing was a useful and legally enforced way to manage email boundaries, they largely perceived it as an impractical, inefficient, and inconvenient method that they would not actually use. Instead, they usually looked for other ways to get rid of unwanted email. 


\section{Ways of Enforcing Boundaries}

Although we did not screen participants based on volume of received email, all participants reported receiving a high volume of email everyday, with a third of the participants receiving over 75 unwanted email messages a day. Yet, unsubscribing was rare. Almost half of the participants deleted the email message immediately after unsubscribing, with one of them hoping it would be auto-deleted. Most participants chose to get rid of unwanted email via approaches other than unsubscribing. Participants were more accustomed to deleting unwanted messages and found it to be the easiest method to reduce unwanted email in the inbox. P7 even remarked that he "... had that knee jerk reaction to swipe left to delete them." Many participants believed that unsubscribing would not work and considered it more convenient to send unwanted email to the trash folder where it would be automatically deleted after a stipulated period of time. P16 reported that it was more efficient and effective to pick and choose emails that were wanted and discard everything else in order to enforce boundaries between wanted and unwanted information.

In cases of extreme annoyance, participants would mark the received email as spam just to get it out of their inbox:

"... my understanding is if you spam something, that will stop those emails coming." - (P8, Male, 60-69)

Marking email as spam thus served as a boundary enforcement technique that participants believed would prevent future unwanted email from appearing in inboxes.

\section{Desire for Firm Boundaries}

Email from specific senders was frequently identified as unwanted when participants no longer maintained the earlier relationship with those senders. Relocating to a different geographic location was most common circumstance that necessitated changes in prior connections:

"I have not been in Minnesota for almost six years, so there's really no need for this email anymore." - (P5, Male, 30-39)

Other unwanted email was caused by making a purchase from a business, resulting in a seemingly never-ending stream of marketing email from that business:

"I think I bought one item from them one time and now I get a billion emails." (P1, Non-binary, 20-29)

Yet, participants grudgingly accepted some commercial email because they wanted to receive content such as special deals, event information, promotional offers, etc. At the same time, participants were annoyed by the frequency of such email:

“... they must send an email about every hour. It's like way too much. So it almost makes me not want to shop there, because they're annoying." - (P2, Female, 60-69)

Participants were averse to email from political campaigns asking for donations even though they were interested in periodic updates that did not ask for money. P4 mentioned that she actually wanted to receive information from political campaigns, but did not like the feeling that emails asking for donations demanded a response from her. In general, P4 did not want to receive any email that demanded action, such as providing product or service feedback.

Often, participants did not remember the context under which they signed up for a given mailing list or did not remember signing up at all. For instance, P8 encountered a landing page that asked him to enter the email address to unsubscribe. The corresponding email message did not include the email address to which it was sent, and P8 could not determine which of his email addresses would be subscribed to the list, if he had indeed subscribed in the first place. Participants tended to characterize such messages that lacked a specific recipient email address as spam.

Social media notifications were a common example of unwanted email that participants wanted to stop. Typically, participants did not understand why they were receiving such notifications:

"I get a lot [of email] from Facebook, and I'm not even on Facebook. But I get a lot of emails from Facebook saying my wife has posted a new photo on Facebook or one of my cousins has posted something on Facebook ... But I never gave Facebook my email address, so I don't even know why I get those." (P8, Male, 60-69)

P8 was annoyed by the blurring of boundaries between social media posts and personal email. Further, he did not appreciate receiving email messages triggered by someone else's posts on another platform.

Other contexts in which participants wanted to establish boundaries were situations where they remembered using a product or service or browsing a website, but did not remember signing up for an email subscription. P3 pointed out that some websites would not permit access to their content without subscribing to a newsletter or creating an account. In such cases, participants were forced to set up an account if they really wanted to access the content.

\section{Insufficient Control over Boundaries}

Consent is paramount for managing boundaries. Participants stated that simply using an organization's products or services did not indicate their permission for the organization to send them irrelevant email. Participants perceived unwanted marketing email as inappropriate and felt betrayal upon receiving such email. P8 pointed out that unwanted subscriptions can result from email addresses being shared among organizations affiliated with each other:

"I get a lot of that kind of stuff that I didn't subscribe to. It's like, 'Well why did I get this?' But somebody has given them my email address." - (P8, Male, 60-69)

P10 believed that the U.S. Postal Service was responsible for distributing his email address for marketing ("I think the post office gave it").

Participants strongly disliked being automatically opted in for list subscriptions:

"... as part of that they have your name, your email, so you've agreed in some way, shape, or form to get promotional [email] in some way because oh, hey, you bought 
this, so let's bombard you with this or that and that's how they got you. And now in order to get off of it, you need to unsubscribe." - (P7, Male, 30-39)

Similarly, P5 felt that his email address was often used without consent:

“They must've secretly added me to some list I wasn't aware of... I was staying in a Hyatt hotel and without asking me they signed me up for all of these promotional emails and consumer feedback emails. I kept getting them for about a week after staying at the hotel ... No one at the front desk asked if I wanted to be signed up for emails at least when I was checking in." - (P5, Male, 30-39)

In the above case, P5 wanted someone (the "front desk") to ask for consent to be added to an online mailing list and did not consider physical presence or use of the service as a providing the organization the permission to use his email address for promotional purposes.

The desire to be asked for consent for email subscriptions conflicts with the nature of the email protocol that lacks explicit gate keepers and access control. To further complicate matters, automatic opt-in made it difficult for participants to distinguish legitimate email from spam. For instance, P16 felt that he could not effectively distinguish between email he had consented to receive versus that which he was forced to receive.

\section{Boundary Turbulence}

Boundary turbulence refers to the problems and breakdowns that arise when the co-owners of information disagree on its use [15]. For example, P10 believed that email is for interpersonal communication and should never be used as a marketing tool, a perspective that commercial entities clearly do not share. Participants in our study spoke of issues that arose when the co-owners of their information, i.e., the senders using their email addresses to contact them, continued to use it in undesired ways.

\section{Lack of Respect for Boundaries}

Sometimes, unsubscribing required participants to click through steps they felt were unnecessary. For instance, P2 encountered a case where she had to verify her information three times to reach the unsubscribe confirmation page. Similarly, unsubscribing from email sent by previously attended educational institutions needlessly imposed a heavy burden by requiring participants to $\log$ in. Such experiences underscore that organizations are not motivated to make it easy to unsubscribe because that would decrease the audience for marketing messages. In fact, participants suspected that organizations intentionally made the processes difficult to find and enact:

"I don't think a business will do that because they don't want to make it that easy to unsubscribe. The whole point is they want you to get those emails. If you ask them to stop, they have to. You have to give that consumer the ability legally, but they don't want to do that. So they're not going to make it easy, almost guarantee you." - (P7, Male, 30-39)
In addition to obscure and convoluted processes, participants often encountered landing pages with messages attempting to persuade them not to unsubscribe. Most participants strongly disliked such messages and found them emotionally manipulative and insincere:

"I really hate when they have stuff like, oh wait, I've changed my mind, I don't wanna unsubscribe. Or, we're sorry to see you go. 'Cause I feel like they're just trying to do some emotional manipulation ... I just hate that." (P4, Female, 30-39)

Social relationships posed another barrier to unsubscribing. For instance, P1 reported remaining subscribed to unwanted email because the subscription was initiated upon request of a professional colleague and unsubscribing could have been perceived as unkind and created conflict. Similarly, P4 found it difficult to stop email from a mailing list because unsubscribing required writing an email message to a person:

"I had avoided trying to unsubscribe because I thought I didn't want to hurt their feelings, or because it's people that I personally spent time with." (P4, Female, 30-39)

\section{Fear of Malicious Actors}

Participant behavior during the process of unsubscribing revealed a number of implicit concerns related to privacy and online safety. One of the major concerns was the legitimacy of the email sender. P9, for example, feared an email message was not legitimate because the images did not load properly. Similarly, P18 was suspicious of email messages with unsubscribe links that were broken or was marked as suspicious:

"I couldn't unsubscribe, because Google identified that as a suspicious link ... generally I don't click on links from spam." - (P18, Female, 20-29)

If $\mathrm{P} 5$ received unwanted email from an unrecognized sender, he checked the domain from which the message was sent and, if needed, verified its legitimacy via searching the web. For example, before interacting with an email message sent from 'facebookmail.com' domain, P5 verified that it was indeed a legitimate email from Facebook.

In one case, P9 encountered an email message containing a link labeled 'Safe Unsubscribe.' Safe Unsubscribe is a third party service that senders of newsletters or commercial email can use to provide one-click unsubscribe functionality ${ }^{2}$. However, the email message included no explanation, making P9 wonder, "What [is] the difference between Safe Unsubscribe and just regular unsubscribe?". She interpreted the 'Safe Unsubscribe' label to imply that other subscription mechanisms were perhaps unsafe.

Fear of malicious senders made it more likely that participants would get rid of spam by deleting, instead of attempting to unsubscribe and disclosing information to potentially malicious actors. When asked to unsubscribe from email in their spam folders, P5 and P7 were reluctant to even click on the email messages from senders they did not recognize. They believed

2 https: //Www . vadesecure. com/en/

vade-retro-safe-unsubscribe-introduction/ 
that the messages were sent to spam for a reason and did not want to bother unsubscribing, instead choosing to delete them:

"It might be a phishing scheme and I don't see any real benefit to even trying to unsubscribe from those if they even give me an option. I'm afraid that by doing so I'm probably just giving them more information than is necessarily." - (P5, Male, 30-39)

Participants were confused and often suspicious of messages from a known service when they appeared to be sent by a person they did not know. P2 felt that email from organizations that appeared to be sent by a person was click-bait because she expected email from individuals to be personal email:

“... I get a lot of emails from people I don't know because I work with prospective students and current students, so people email me, so I always open up the email, especially if it's just a person's name." - (P2, Female, 60-69)

$\mathrm{P} 2$ reported being confused and annoyed upon realizing that the 'person' sending her email was actually a marketing campaign. Others believed that such email is designed to scam the recipient and elicit information:

“... if somebody ... started sending [email that] looked like it was an email from this person ... I went, wait, is this [a particular] person? ... I think they're scams to try to get you to succumb so that you will give them information. - (P6, Female, 60-69)

\section{Boundary Violations}

Boundary violations occurred when someone made an attempt to assert a boundary by expressing the intention to unsubscribe, but the sender continued to send email or ask for additional information. When participants were trying to establish a boundary by unsubscribing, they did not expect to be asked for more information or receive more email from that sender. For example, P2 encountered a case where unsubscribing required filling out personal information like name, address, and email address, leaving her confused about the need for the information given her desire to stop further communication.

Unsubscribing is often accomplished through third party systems that present landing pages requesting feedback from those who unsubscribe. The older participants, P6 and P8, were willing to provide a reason for unsubscribing, but most others were annoyed about being asked to input information.

Upon unsubscribing, especially from mailing lists, many participants received email messages confirming that they had unsubscribed which seemed to defeat the purpose of unsubscribing. Once unsubscribed, participants were “... usually past whatever phase in my life that I don't need that service or product anymore" (P1). While participants wanted and appreciated a clear confirmation during the unsubscribing process, they did not want additional email after unsubscribing.

\section{IMPLICATIONS}

The findings of our study illustrate that poor implementation of unsubscribing mechanisms prevents users from establishing desired email boundaries. Providing users with more effective support for stopping unwanted email would help them regain some autonomy when dealing with commercial and institutional email. To that end, we offer several suggestions for improving the user experience and effectiveness of the unsubscribing process in a variety of ways. These recommendations are based on ideas mentioned by the participants during the study and captured in the data analysis processes. Where needed, we have included additional detail to make the recommendations actionable.

Misalignment between business interests of companies and desires of users may mean a lack of sufficient incentive for commercial entities to act upon the recommendations we offer below. Nevertheless, we argue that it is valuable to document these ideas. We have left some of the suggestions intentionally broad in order to facilitate further discussion on feasible concrete implementation. It may be that companies can find ways to provide users with greater email boundary management by addressing some of these issues in a manner synergistic with broader business considerations.

\section{Standardize the Process of Unsubscribing}

Even in our relatively small scale investigation, participants encountered a large variety of mechanisms for unsubscribing. While some processes were common across several senders due to the use of the same third party services, others were custom and typically did not follow consistent conventions. In the most egregious cases, the unsubscribe process required mandatory disclosure of personal information or performance of steps unnecessary to fulfill the unsubscribe request. Developing a standard set of design patterns for the user interaction of the unsubscribe process can help achieve the consistency and familiarity that users seek. Standards for collaboration that ensure appropriate organizational communication and coordination among employees who craft the email messages and developers who design and implement the unsubscribe functionality can help as well.

In addition, email platforms could facilitate unsubscribing from unwanted email by leveraging computational capabilities and/or automate one or more parts of the unsubscribe process. Many email clients already inspect email messages for an unsubscribe link and prominently present it separately from the body of the message. Such a functionality could be enhanced by incorporating a "one click" unsubscribe button that automatically handles the interaction of the unsubscribe process behind the scenes. Development of standards could help increase interoperability across email platforms with the potential for implementing a common Application Programming Interface (API) for automated unsubscribing. Such automation can help fulfill the desire expressed by participants to achieve unsubscription with the same efficiency as deleting email messages or flagging spam, which can be performed in bulk by selecting multiple messages. While the third party service unroll.me $\mathrm{m}^{3}$ offers similar functionality, it requires that users grant it access to their inboxes, thus compromising users' privacy. Incorporating automated unsubscription capabilities into the email clients avoids exposing email to third parties for these purposes.

\footnotetext{
${ }^{3}$ https://unroll.me
} 
In addition, automation could be applied to implement tracking mechanisms such that unsubscriptions are monitored by the email client to ensure that the respective senders have stopped sending the unwanted email messages. Several participants noted a desire to track future email to ensure that the unwanted email messages did indeed stop. Such tracking could be implemented as an additional feature within email clients, thus relieving users of remembering each unsubscribe request and being on the lookout for a violation. Once users establish a boundary by unsubscribing, they need to be assured that the other party will continue to respect that boundary.

\section{Follow Good User Interface Design Principles}

Several of the frustrations experienced by the participants could be addressed by improving the user interface of the landing page reached by clicking on the unsubscribe link. Ideally, a landing page should ask for nothing more than a confirmation of the intent to unsubscribe in case a user arrived at the page due to an accidental click. Moreover, the text should be worded concisely and clearly to avoid confusing users about whether the selections lead to opt-out. The buttons on the landing page should provide only the options to confirm and cancel the unsubscribe request. Seeking mandatory additional input, such as reasons for unsubscribing and email address for confirmation, should be avoided. While such input is an avenue for companies to solicit feedback, it should be optional because users are typically uninterested in extra steps beyond clicking the unsubscribe link to opt out. Similar attention to wording and feedback is necessary for the unsubscription confirmation page reached via the landing page. The confirmation page should help users confirm quickly and confidently that their unsubscribe request was successful.

User interface improvements to email messages would make it easier for users to assert email boundaries. Better formatting can make it easier to identify the unsubscribe link within a message and signal the consequences of clicking the link. Such signals help set expectations regarding the likely result of clicking the link, such as going to a webpage, composing a new email message, etc. Email messages could include additional relevant information such as a clear indication of the email address to which the message was sent, a snippet describing why the user is receiving the email, etc. Apart from helping users, such transparency could help senders establish legitimacy and trust and avoid being characterized as a spammer or scammer. In addition, unsubscribing mechanisms should avoid user experiences that break expectations and resemble strategies used by malicious actors, such as surprising page redirects or non-standard URLs.

\section{Allow Users to Set Clear Boundaries}

Participants wanted to be explicitly asked to sign up for commercial email. Further, participants wanted their consent to apply to only the specific context in which it was given, and not be treated as a free pass to sell their information to third parties. Appropriate consent mechanisms can avoid situations where users are unable to recall signing up for a specific email message. Participants wanted to know what to expect after consenting to an email subscription. To that end, user interfaces for email subscriptions should be designed such that users have the ability to choose the content of interest along with an acceptable frequency for receiving the content.

Participants found it difficult to avoid receiving large amounts of unwanted email in order to receive email of relevance to their interests and needs. For instance, some participants wanted email about promotional offers but could not sign up to receive just that information without the various other unwanted email messages from the respective companies. Providing granular subscription choices can help senders fulfill specific user needs without bombarding them with unwanted email in the process. In this regard, senders need to be precise about the types of email messages connected to specific subscriptions. For instance, senders should clearly indicate whether an unsubscribe request would result in being unsubscribed from all communication, including updates on purchases, or just from specific types of email messages. Without such clarity, users remain apprehensive that an unsubscribe request may accidentally lead to missing something of importance.

Privacy legislation often includes regulations regarding communication boundaries that commercial entities must respect. For example, the European Union's General Data Protection Regulation (GDPR) [20] requires consumers to opt in to receive marketing communication and permit sharing of their personal information, including email addresses, with third parties. Laws and reglations in the United States typically do not prohibit initial opt-in without consent but do generally require that commercial entities provide a clear opt-out and respect the wishes of those who avail of the option. The U.S. CAN-SPAM Act of 2003 mandates that senders honor opt-out requests, but in many instances, the participants in our study believed that their requests were processed too slowly or not honored at all. Additional laws and regulations specifically targeted at unsubscription could perhaps be used as a vehicle to incentivize standardization and facilitate more effective enforcement.

\section{CONCLUSION}

Users struggle to manage unwanted email, leaving them with a sense of decreasing control over their inboxes. Even though participants in our study were relatively familiar with the process of unsubscribing, user experience issues prevented effective use of available mechanisms to establish and assert effective email boundaries. The barriers to unsubscribing uncovered in our study underscore the need for increased attention by commercial entities and email providers to making unsubscribing truly functional for end users.

\section{ACKNOWLEDGMENTS}

This work was supported by an endowment from AT\&T to Michigan State University. We thank study participants and those who helped pilot the study procedures. We acknowledge anonymous reviewers for providing feedback that helped refine the paper.

\section{REFERENCES}

[1] Irwin Altman. 1976. A Conceptual Analysis. Environment and Behavior 8, 1 (1976), 7-29. DOI : http://dx.doi.org/10.1177/001391657600800102 
[2] Frank Bentley, Nediyana Daskalova, and Nazanin Andalibi. 2017. "If a Person is Emailing You, It Just Doesn't Make Sense": Exploring Changing Consumer Behaviors in Email. In Proceedings of the 2017 CHI Conference on Human Factors in Computing Systems (CHI '17). Association for Computing Machinery, New York, NY, USA, 85-95. DOI :

http://dx.doi.org/10.1145/3025453.3025613

[3] Nediyana Daskalova, Frank R. Bentley, and Nazanin Andalibi. 2017. It's All About Coupons: Exploring Coupon Use Behaviors in Email. In Proceedings of the 2017 CHI Conference Extended Abstracts on Human Factors in Computing Systems (CHI EA '17). Association for Computing Machinery, New York, NY, USA, 1152-1160. DOI :

http://dx.doi.org/10.1145/3027063.3053339

[4] Dotan Di Castro, Zohar Karnin, Liane Lewin-Eytan, and Yoelle Maarek. 2016. You've Got Mail, and Here is What You Could Do With It! Analyzing and Predicting Actions on Email Messages. In Proceedings of the Ninth ACM International Conference on Web Search and Data Mining (WSDM '16). Association for Computing Machinery, New York, NY, USA, 307-316. DOI : http://dx.doi.org/10.1145/2835776.2835811

[5] K. Anders Ericsson and Herbert A. Simon. 1980. Verbal Reports as Data. Psychological Review 87, 3 (1980), 215-251. DOI :

http://dx.doi.org/10.1037/0033-295X.87.3.215

[6] Kim Flaherty. 2018. Top 10 Design Mistakes in the Unsubscribe Experience. (Apr 2018). Retrieved January 7, 2020 from

https://www.nngroup. com/articles/unsubscribe-mistake.

[7] Iftah Gamzu, Liane Lewin-Eytan, and Natalia Silberstein. 2018. Unsubscription: A Simple Way to Ease Overload in Email. In Proceedings of the Eleventh ACM International Conference on Web Search and Data Mining (WSDM '18). Association for Computing Machinery, New York, NY, USA, 189-197. DOI : http://dx.doi.org/10.1145/3159652.3159698

[8] Khim-Yong Goh, Kai-Lung Hui, and Ivan P. L. Png. 2015. Privacy and Marketing Externalities: Evidence from Do Not Call. Management Science 61, 12 (2015), 2982-3000. DOI :

http://dx.doi.org/10.1287/mnsc. 2014.2051

[9] Catherine Grevet, David Choi, Debra Kumar, and Eric Gilbert. 2014. Overload is Overloaded: Email in the Age of Gmail. In Proceedings of the SIGCHI Conference on Human Factors in Computing Systems (CHI '14). Association for Computing Machinery, New York, NY, USA, 793-802. DOI :

http://dx.doi.org/10.1145/2556288.2557013

[10] The Radicati Group. 2019. Email Statistics Report 2015-2019. (2019). Retrieved January 07, 2020 from https: //www.radicati.com/wp/wp-content/uploads/2015/02/ Email-Statistics-Report-2015-2019-Executive-Summary . pdf.

[11] Greg Guest, Arwen Bunce, and Laura Johnson. 2006. How Many Interviews Are Enough?: An Experiment with Data Saturation and Variability. Field Methods 18, 1 (2006), 59-82. DOI :

http://dx.doi.org/10.1177/1525822X05279903

[12] Il-Horn Hann, Kai-Lung Hui, Sang-Yong T. Lee, and Ivan P. L. Png. 2008. Consumer Privacy and Marketing Avoidance: A Static Model. Management Science 54, 6 (2008), 1094-1103. DOI :

http://dx.doi .org/10.1287/mnsc. 1070.0837

[13] Pedro Leon, Blase Ur, Richard Shay, Yang Wang, Rebecca Balebako, and Lorrie Cranor. 2012. Why Johnny Can't Opt out: A Usability Evaluation of Tools to Limit Online Behavioral Advertising. In Proceedings of the SIGCHI Conference on Human Factors in Computing Systems (CHI '12). Association for Computing Machinery, New York, NY, USA, 589-598. DOI : http://dx.doi.org/10.1145/2207676.2207759

[14] Stephen T. Margulis. 2011. Three Theories of Privacy: An Overview. In Privacy Online: Perspectives on Privacy and Self-Disclosure in the Social Web, Sabine Trepte and Leonard Reinecke (Eds.). Springer Berlin Heidelberg, Berlin, Heidelberg, 9-17. DOI : http://dx.doi .org/10.1007/978-3-642-21521-6_2

[15] Sandra Petronio. 2002. Boundaries of Privacy: Dialectics of Disclosure. State University of New York Press.

[16] Emilee Rader and Anjali Munasinghe. 2019. "Wait, Do I Know This Person?": Understanding Misdirected Email. In Proceedings of the 2019 CHI Conference on Human Factors in Computing Systems (CHI '19). Association for Computing Machinery, New York, NY, USA, Article Paper 290, 13 pages. DOI:

http://dx.doi.org/10.1145/3290605.3300520

[17] Jacquelyn Trussell. 2004. Is the CAN-SPAM Act the Answer to the Growing Problem of Spam. Loyola Consumer Law Review 17516 (2004), 175-188. Issue 2. Available at:

http://lawecommons. luc. edu/lclr/vol16/iss2/5.

[18] United States Congress. 2003. Controlling the Assault of Non-Solicited Pornography and Marketing Act of 2003 (CAN-SPAM Act). (2003).

[19] Hal Varian, Fredrik Wallenberg, and Glenn Woroch. 2005. The Demographics of the Do-Not-Call List. IEEE Security \& Privacy 3, 1 (Jan 2005), 34-39. DOI : http://dx.doi.org/10.1109/MSP.2005.28

[20] Paul Voigt and Axel Von dem Bussche. 2017. The EU General Data Protection Regulations (GDPR): A Practical Guide. Springer, Cham. DOI : http://dx.doi.org/10.1007/978-3-319-57959-7 
[21] Steve Whittaker and Candace Sidner. 1996. Email Overload: Exploring Personal Information Management of Email. In Proceedings of the SIGCHI Conference on Human Factors in Computing Systems (CHI '96).
Association for Computing Machinery, New York, NY, USA, 276-283. DOI:

http://dx.doi.org/10.1145/238386.238530 